\title{
Technical Report on Ab Initio Studies of Coke Formation on Ni Catalysts During
}

Methane Reforming

Annual Technical Report for annual period ending 9/25/03

Principal author: David S. Sholl

DOE Award Number. DE-FG26-02NT41538

Submitting institution: Carne gie Mellon University, Pittsburgh, PA 15213 


\section{Disclaimer}

This report was prepared as an account of work sponsored by an agency of the United States Government. Neither the United States Government nor any agency thereof, nor any of their employees, makes any warranty, express or implied, or assumes any legal liability or responsibility for the accuracy, completeness, or usefulness of any information, apparatus, product, or process disclosed, or represents that its use would not infringe privately owned rights. Reference herein to any specific commercial product, process, or service by trade name, trademark, manufacturer, or otherwise does not necessarily constitute or imply its endorsement, recommendation, or favoring by the United States Government or any agency thereof. The views and opinions of authors expressed herein do not necessarily state or reflect those of the United States Government or any agency thereof. 


\begin{abstract}
The atomic-scale processes that control the formation of carbon deposits on Nicatalysts in reforming applications are poorly understood. Ab initio Density Functional Theory calculations have been used to examine several key elementary steps in the complex network of chemical reactions that precedes carbon formation on practical catalysts. Attention has been focused on the disproportionation of CO. A comparative study of this reaction on flat and stepped crystal planes of $\mathrm{Ni}$ has provided the first direct evidence that surface carbon formation is driven by elementary reactions occurring at defect sites on $\mathrm{Ni}$ catalysts.
\end{abstract}




\section{Table of Contents}

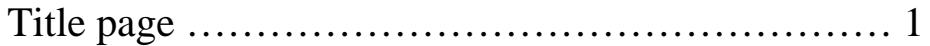

Disclaimer ......................................2

Abstract ........................................... 3

Table of Contents .................................4

Executive summary ...............................5

Results and discussion .............................6

Conclusion ......................................... 7

References ..................................... 7 


\section{Executive Summary}

Reforming of methane with either water (steam reforming) or $\mathrm{CO}_{2}$ (dry reforming) to produce syngas is a significant pathway for converting natural gas into more versatile chemical feedstocks. A significant difficulty with practical use of $\mathrm{Ni}$ as a catalyst for methane reforming is the formation of surface carbon (coke), which leads to catalyst deactivation. We have used quantum chemistry calculations, specifically, plane wave Density Functional Theory (DFT), to elucidate the atomic-scale mechanisms for the initial stages of carbon deposition on $\mathrm{Ni}$ surfaces. One important elementary reaction leading to the net formation of surface carbon is the disproportionation of adsorbed $\mathrm{CO}$. We have used DFT to examine the adsorption of $\mathrm{CO}, \mathrm{C}$, and $\mathrm{O}$ on a set of Ni surfaces chosen to represent both the atomically flat low Miller index surfaces likely to exist on practical catalysts and also surface defect sites that are created by surface steps. Specifically, we have examined $\mathrm{Ni}(111), \mathrm{Ni}(110), \mathrm{Ni}(210)$, and $\mathrm{Ni}(531)$. The latter two surfaces are stepped surfaces which, in their ideally terminated structure, are well suited for representing general classes of surface defects within the limitations of plane wave DFT.

Our calculations for adsorbed CO yield structural data in excellent agreement with experimental results where these are available. More importantly, our results confirm earlier experimental calorimetry data that indicated that the adsorption energy of $\mathrm{CO}$ on $\mathrm{Ni}$ is not surface sensitive. That is, the binding energies on a range of flat and stepped surfaces are quite similar. The adsorption of the relevant atomic species, $\mathrm{C}$ and $\mathrm{O}$, however, is found to be structure sensitive, with adsorption of these species near surface steps being considerably more favorable than on flat surfaces. This observation points to surface defects as potentially playing a key role in the initial nucleation of surface carbides on Ni catalysts.

While understanding the thermochemistry of adsorbed species gives some insight into surface reaction mechanisms, a much more complete picture can be obtained by rigorously determining the transition states between reactants and products for reactions of interest. Estimates of the energy of the transition state for $\mathrm{CO}$ disproportionation were obtained using the semi-empirical unity bond index-quadratic exponential potential (UBI QEP) method. This method estimates transition state energies using only information about the reactant and product states. These estimates suggest that $\mathrm{CO}$ disproportionation is quite unfavorable on flat $\mathrm{Ni}$ surfaces relative to molecular desorption of $\mathrm{CO}$, but that disproportionation is more likely to occur on stepped surfaces. To understand the accuracy of these estimates, calculations have been performed to rigorously locate the transition states related to $\mathrm{CO}$ disproportionation on $\mathrm{Ni}(111)$. Our calculations show that the $\mathrm{C}$ and $\mathrm{O}$ atoms move through a series of local minima separated by transition states as the atoms become separated on the surface until the limit of well separated atoms used as the final state in the UBI-QEP estimate is reached. Significantly, the true transition state for this process is substantially higher in energy than the UBI-QEP estimate.

During methane reforming on $\mathrm{Ni}$ catalysts, species containing $\mathrm{C}, \mathrm{O}$, and $\mathrm{H}$ are all simultaneously present on the catalyst surface. Even for the small molecules relevant to this process, the number of distinct elementary reactions that needs to be considered to completely describe practical catalysis is enormous. To move towards a more complete description of these systems, we have used DFT to examine the adsorption of $\mathrm{H}$ on the same $\mathrm{Ni}$ surfaces mentioned above. Atomic $\mathrm{H}$ can adsorb both on top of metal surfaces 
and also in subsurface interstitial sites, so both modes of adsorption are being considered in our calculations. Our calculations provide a comprehensive picture of the mobility and binding energies of atomic $\mathrm{H}$ on flat and stepped Ni surfaces.

\section{Results and Discussion}

Spin polarized plane wave density functional theory (DFT) calculations were performed with the Vienna $a b$ initio Software Package (VASP), which uses ultrasoft pseudopotentials to treat ion-electron interactions. VASP is known to produce results in close agreement with other plane wave DFT codes. All calculations were performed using the generalized gradient approximation with the PW91 exchange correlation functional. The energy cutoff for all calculations was $396 \mathrm{eV}$, which is the cutoff used in generating the oxygen pseudopotentials. The Monkhorst-Pack method was used to generate $k$-points. Calculations on the Ni(111), (110), (210), and (531) surfaces used $6 \times 6 \times 2,8 \times 8 \times 2,5 \times 5 \times 1$, and $5 \times 5 \times 1 k$-points, respectively. These methods predict the bulk lattice constant and magnetic moment for $\mathrm{Ni}$ in good agreement with experimental values. In our surface calculations, the surface unit cells were defined using the DFT optimized Ni lattice constant and a vacuum spacing greater than $11 \AA$ was used for each system. We did not apply dipole corrections to our calculations. The thickness of each surface slab was chosen to be at least as thick as a three layer Ni(111) slab. Our previous calculations showed that using slabs with this thickness predicts the properties of adsorbed $\mathrm{CO}$ in excellent agreement with equivalent calculations with thicker slabs. Unless otherwise noted, all calculations of adsorbate properties were performed with a single adsorbed atom in the computational supercell. To examine the impact of coverage on adsorption properties, we varied the number of surface unit cells in the computational volume. The adsorbed $\mathrm{C}$ and $\mathrm{O}$ were placed on one side of the slab only, and all atoms except the lowest layer of $\mathrm{Ni}$ atoms were allowed to fully relax. This procedure cannot provide information on the complex reconstructions that have been reported experimentally for $\mathrm{C}$ adsorption on $\mathrm{Ni}(111)$ and $\mathrm{Ni}(110)$, since the configurations in our calculations are restricted to the symmetry of the computational supercell, and more importantly, it is likely that energy barriers must be surmounted before these reconstructions can take place. We restrict our attention to $\mathrm{C}$ and $\mathrm{O}$ atoms adsorbed on top of each surface we examine, although we note that presence of subsurface $\mathrm{C}$ has been implicated in some of the surface reconstructions observed experimentally on low index Ni surfaces.

The adsorption energy of an atomic species on each surface was defined by $\mathrm{E}_{\mathrm{ads}}=$ $\mathrm{E}_{a}+\mathrm{E}_{\mathrm{slab}}-\mathrm{E}_{a / \mathrm{slab}}$, where the three terms on the right hand side are the energy of an isolated spin polarized atom, the energy of the bare Ni surface, and the energy of the slab with the adsorbed atom present, respectively. With this definition, a positive value for $\mathrm{E}_{\mathrm{ads}}$ corresponds to a stable adsorption.

The principal result from our calculations with $\mathrm{CO}, \mathrm{C}$, and $\mathrm{O}$ adsorption on $\mathrm{Ni}(111)$, $\mathrm{Ni}(110), \mathrm{Ni}(210)$, and $\mathrm{Ni}(531)$ is that the $\mathrm{CO}$ binding energy is relatively insensitive to the identity of the crystal plane onto which it is adsorbed, but the atomic species are senstitive to the surface structure. The stepped surfaces, $\mathrm{Ni}(531)$ and $\mathrm{Ni}(210)$ exhibit higher binding energies for the atomic species than the flat surfaces, pointing to the importance of defects such as step edges as dominant adsorption sites for these species on real catalysts. A paper describing these results is currently in preparation [1]. 
While understanding the thermochemistry of adsorbed species gives some insight into surface reaction mechanisms, a much more complete picture can be obtained by rigorously determining the transition states between reactants and products for reactions of interest. Estimates of the energy of the transition state for $\mathrm{CO}$ disproportionation were obtained using the semi-empirical unity bond index-quadratic exponential potential (UBI QEP) method. This method estimates transition state energies using only information about the reactant and product states. These estimates suggest that $\mathrm{CO}$ disproportionation is quite unfavorable on flat $\mathrm{Ni}$ surfaces relative to molecular desorption of $\mathrm{CO}$, but that disproportionation is more likely to occur on stepped surfaces. To understand the accuracy of these estimates, calculations have been performed to rigorously locate the transition states related to $\mathrm{CO}$ disproportionation on $\mathrm{Ni}(111)$. Our calculations show that the $\mathrm{C}$ and $\mathrm{O}$ atoms move through a series of local minima separated by transition states as the atoms become separated on the surface until the limit of well separated atoms used as the final state in the UBI-QEP estimate is reached. Significantly, the true transition state for this process is substantially higher in energy than the UBI-QEP estimate. We are currently applying rigorous methods to locate transition states for $\mathrm{CO}$ disproportionation on stepped Ni surfaces within plane wave DFT. This procedure is necessary because the UBI-QEP technique can only be expected to provide qualitative information.

In addition to examining $\mathrm{CO}, \mathrm{C}$, and $\mathrm{O}$ adsorption on $\mathrm{Ni}$, we have applied DFT to examine the adsorption of atomic $\mathrm{H}$ on the same set of four $\mathrm{Ni}$ surfaces. $\mathrm{H}$ can occupy interstitial sites in many metals, so we have examined a variety of subsurface binding sites in addition to the surface binding sites similar to those expected for $\mathrm{C}$ and $\mathrm{O}$. Our calculations support the concept that the diffusion barriers for motion of atomic $\mathrm{H}$ on $\mathrm{Ni}$ surfaces are low relative to other adsorbed species, so hydrogen can be treated in microkinetic models of realistic catalysts as a species that rapidly equilibrates under process conditions. A paper describing these results is currently in preparation [2].

\section{Conclusion}

Plane wave Density Functional Theory calculations have been used to probe the adsorption and reactivity of $\mathrm{CO}, \mathrm{C}, \mathrm{O}$, and $\mathrm{H}$ on a variety of flat and stepped $\mathrm{Ni}$ surfaces. Calculations of this type predict structural and energetic quantities that are in excellent agreement with experimental data when this data is available. Crucially, calculations of this type provide a means to directly examine the relative reactivity of adsorption sites with varying local coordination, a task that is extremely challenging experimentally. Our calculations support the idea that surface defects play an extremely important role in the initial formation of surface carbon on nickel catalysts exposed to CO.

\section{References}

1. First Principles Study of CO dissociation on flat and stepped Ni surfaces Tao Li, Bhawna Bhatia, and David S. Sholl, in preparation for Phys. Rev. B.

2. H Adsorption and Diffusion on Flat and Stepped Ni Surfaces Bhawna Bhatia and David S. Sholl, in preparation for Surf. Sci. 